Article

\title{
Frequency Feature Learning from Vibration Information of GIS for Mechanical Fault Detection
}

\author{
Yang Yuan ${ }^{+} \mathbb{C}$, Suliang $\mathrm{Ma}^{\dagger}$, Jianwen $\mathrm{Wu}^{*}$, Bowen Jia, Weixin Li and Xiaowu Luo \\ School of Automation Science and Electrical Engineering, Beihang University, Beijing 100191, China; \\ sy1703415@buaa.edu.cn (Y.Y.); masuliang@buaa.edu.cn (S.M.); jiabowen109@126.com (B.J.); \\ 13031041@buaa.edu.cn (W.L.); humourluo@163.com (X.L.) \\ * Correspondence: wujianwen@buaa.edu.cn; Tel.: +86-10-8233-8384 \\ + These authors contributed equally to this work.
}

Received: 8 March 2019; Accepted: 23 April 2019; Published: 25 April 2019

\begin{abstract}
The reliability of gas insulated switchgear (GIS) is very important for the safe operation of power systems. However, the research on potential faults of GIS is mainly focused on partial discharge, and the research on the intelligent detection technology of the mechanical state of GIS is very scarce. Based on the abnormal vibration signals generated by a GIS fault, a fault diagnosis method consisting of a frequency feature extraction method based on coherent function (CF) and a multi-layer classifier was developed in this paper. First, the Fourier transform was used to analyze the differences and consistency in the frequency spectrum of signals. Secondly, the frequency domain commonalities of the vibration signals were extracted by using $\mathrm{CF}$, and the vibration characteristics were screened twice by using the correlation threshold and frequency threshold to further select the vibration features for diagnosis. Then, a multi-layer classifier composed of two one-class support vector machines (OCSVMs) and one support vector machine (SVM) was designed to classify the faults of GIS. Finally, the feasibility of the feature extraction method was verified by experiments, and compared with other classification methods, the stability and reliability of the proposed classifier were verified, which indicates that the fault diagnosis method promotes the development of an intelligent detection technology of the mechanical state in GIS.
\end{abstract}

Keywords: gas insulated switchgear; mechanical fault diagnosis; coherent coefficient; one-class support vector machine; support vector machine

\section{Introduction}

As a piece of control and protection equipment in power system [1,2], gas insulated switchgear (GIS) plays a significant role in high-voltage power grids. Discovering potential defects and hidden danger in the process of operation of GIS equipment in time can ensure the reliability and security of power grid operations.

The existing research on the reliability of GIS is mainly focused on the insulation fault diagnosis through signal analysis, and many experts have conducted extensive research on this topic. The main detection methods of partial discharge defects include the electrical method [3-5], acoustic method [6,7] and chemical method [8,9]. Aiming at the condition monitoring and diagnosis of gas insulated structures, a real-time measurement system combining signal acquisition, mode generation, feature extraction and defect recognition was proposed [10]. The ultra high frequency (UHF) method was used to analyze the characteristics of partial discharge, and short-time Fourier transform (STFT) [11] was used to describe the time-frequency characteristics [12,13]. Combined weight function classification tools and K-means clustering, and pulse parameters in both time and frequency domains were used to effectively identify noise signals and discharge pulses [14]. 
Compared with insulation faults, the development of intelligent diagnosis technology for mechanical faults in GIS is very slow. Under the action of electromotive force generated by AC current in conductors, the vibration signal in the fault changes correspondingly compared with the normal situation. In order to realize the intelligent diagnosis of mechanical faults in GIS, it is necessary to study in depth the characteristics of vibration signals of the GIS shell. The empirical mode decomposition (EMD) [15] method was used to analyze the vibration signal, and the characteristic matrix was defined to form the criterion of mechanical fault in GIS [16]. The full-acoustic acquisition method was used to collect different mechanical fault data, and the acoustic characteristics of signal was summarized to conduct fault diagnosis [17]. The transient vibration characteristics of GIS were analyzed by using finite element simulation software ANSYS, and the theoretical basis of mechanical defect detection technology in GIS based on vibration information was provided $[18,19]$. The vibration mechanism of GIS was studied in depth, and by extracting features of vibration signals using spectrum analysis, a method for detecting the mechanical state of GIS based on vibration information was proposed [20,21]. A new algorithm, which is composed of the k-nearest neighbor algorithm and the fuzzy c-means clustering algorithm, for the mechanical fault diagnosis of ultra-high voltage GIS was proposed to realize the detection of the mechanical state of GIS [22].

Generally speaking, the aforementioned documents have made great contributions to the development of mechanical fault diagnosis technology in GIS. However, due to the non-linearity, signal dispersion and noise interference of the GIS system, it is difficult to extract features and screen feature space. The features extracted from the aforementioned documents are insufficient, and the problem is more prominent when the number of samples is large. In addition, the training process of a single classifier is affected by the overall error rate, so the model may favor the majority class and ignore the minority class. The feature extraction method based on coherent function (CF) [23] can summarize the similarities of a spectrum and get the feature sets, and the union of all typical fault feature sets is selected as the feature atlas of GIS fault description. Holistic learning [24,25] is very common in machine learning [26,27]. A series of single weak classifiers are constructed and combined to classify or predict new data by a weighted or unweighted voting method. One-class support vector machines (OCSVM) [28-30] can solve the problem of unbalance between normal data and fault data; beyond this, it can judge the unknown faults, and the feature has been applied in the field of fault diagnosis. For example, system combining model-based diagnosis and data-driven anomaly classifiers for fault isolation used OCSVM to identify unknown faults, and the validity of the method was verified in the internal combustion engines [31,32]. SVM $[33,34]$ can effectively divide the feature space and better classify the fault conditions.

In this paper, a new feature extraction method based on CF was proposed, and a multi-layer classifier composed of OCSVM and support vector machine (SVM) was constructed. The feasibility of the feature extraction method was verified by experiments, and the advantages of the proposed classifier were verified by comparing with the general classification methods such as Softmax [35], SVM, back propagation neural networks (BPNN) [36] and naive Bayes (NB) [37]. The main contributions of this paper can be summarized as follows:

(1) GIS mechanical fault is diagnosed by a holistic approach which integrates the vibration signal acquisition system, feature extraction based on CF and a multi-level classifier composed of OCSVMs and SVM;

(2) The $\mathrm{CF}$ is introduced into the feature screening process, and a method of feature extraction based on CF with double thresholds is proposed, which provides a new idea for feature screening and can fully describe characteristics of the vibration signal;

(3) A multi-layer classifier composed of OCSVM and SVM is designed to diagnose GIS faults.

The remainder of this paper is organized as follows: Section 2 introduces the vibration information acquisition system of GIS, the experiment platform and the vibration signal analysis; Section 3 presents the method of extracting vibration features; Section 4 discusses the establishment of GIS fault classifier; 
Section 5 discusses the parameters of the diagnosis model and the optimization of feature space, and compares the method with other traditional diagnosis methods to prove the improvement in the diagnosis accuracy. Finally, Section 6 summarizes the contributions of the paper.

\section{Experiments and Vibration Data Analysis}

\subsection{Experiments}

In order to collect the data needed for the study of mechanical fault detection technology in GIS, a YD-81D acceleration sensor, DHF-7-3 charge amplifier and NI PCI-4472B acquisition card were used to build the vibration signal acquisition system, and the parameters of the system are shown in the Table 1.

Table 1. Parameters of the acquisition system.

\begin{tabular}{cc}
\hline Parameters & Value \\
\hline measuring range $(\mathrm{g})$ & \pm 0.5 \\
sensitivity $(\mathrm{V} / \mathrm{g})$ & 10 \\
maximum output voltage $(\mathrm{V})$ & \pm 5 \\
weight of a sensor $(\mathrm{g})$ & 10 \\
sampling rate $(\mathrm{kHz})$ & 10 \\
sampling time length $(\mathrm{ms})$ & 100 \\
\hline
\end{tabular}

The experimental object is a $110 \mathrm{kV}$ three-phase common-box GIS experimental platform, as shown in Figure 1a. The types of faults simulated in this paper are shown in Figure 1b: (1) Isolation switch fault - the fault can be simulated by adjusting the position of the isolation switch; (2) Looseness of flange screw - the fault can be simulated by loosening three bolts used to fix flange; (3) Looseness of stone bolt-the fault can be simulated by loosening two bolts supporting GIS in section A. The experimental data recorded are shown in Table 2. The vibration data of each of the working conditions were collected 200 times with the alternating current $(\mathrm{AC})$ of $50 \mathrm{~Hz}$ and $1000 \mathrm{~A}$, forming the data set of the research in this paper.
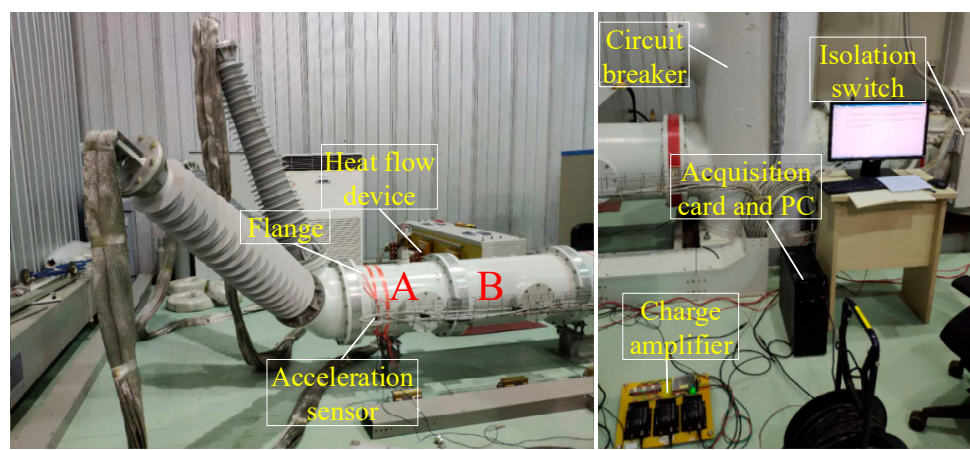

(a) Experiment and field testing
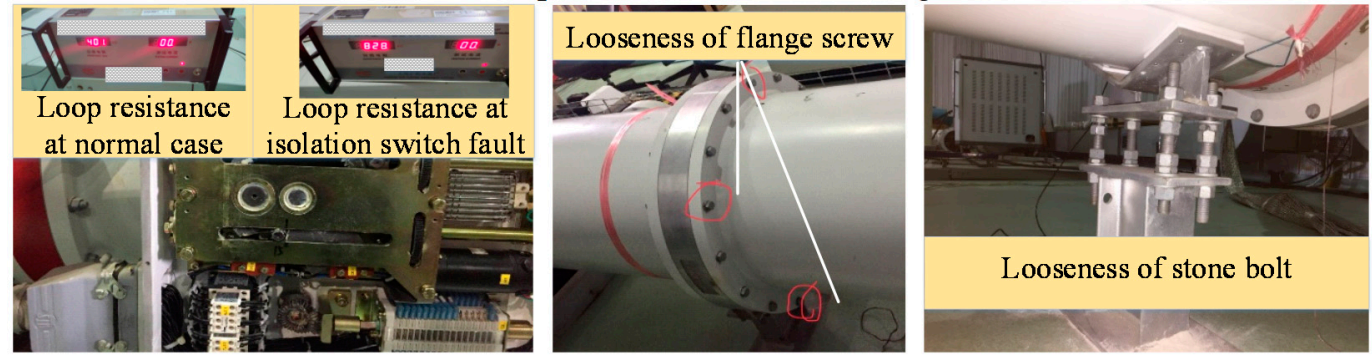

(b) Mechanical fault simulation

Figure 1. Experiment and mechanical fault simulation. 
Table 2. Summary of states of gas insulated switchgear (GIS) considered in this study.

\begin{tabular}{cccc}
\hline Health Condition & Category Label & Description of State & Data Illustrate \\
\hline Healthy & Class 1 & Normal case & $200 \times 4$ groups of GIS \\
& Class 2 & Isolation switch fault & vibration data were \\
False & Class 3 & Looseness of flange screw & collected under 1000 A \\
& Class 4 & Looseness of stone bolt & current and four classes \\
\hline
\end{tabular}

\subsection{Vibration Data Analysis}

When AC flows into the conductor, the vibration information shows strong periodicity. The reason is that the excitation source of the GIS vibration is electromagnetic force, of which the frequency is twice that of the AC frequency [16]. Then, the traditional Fourier transform can be used to analyze the amplitude-frequency distribution characteristics of the vibration signal, as showed in Figure 2.
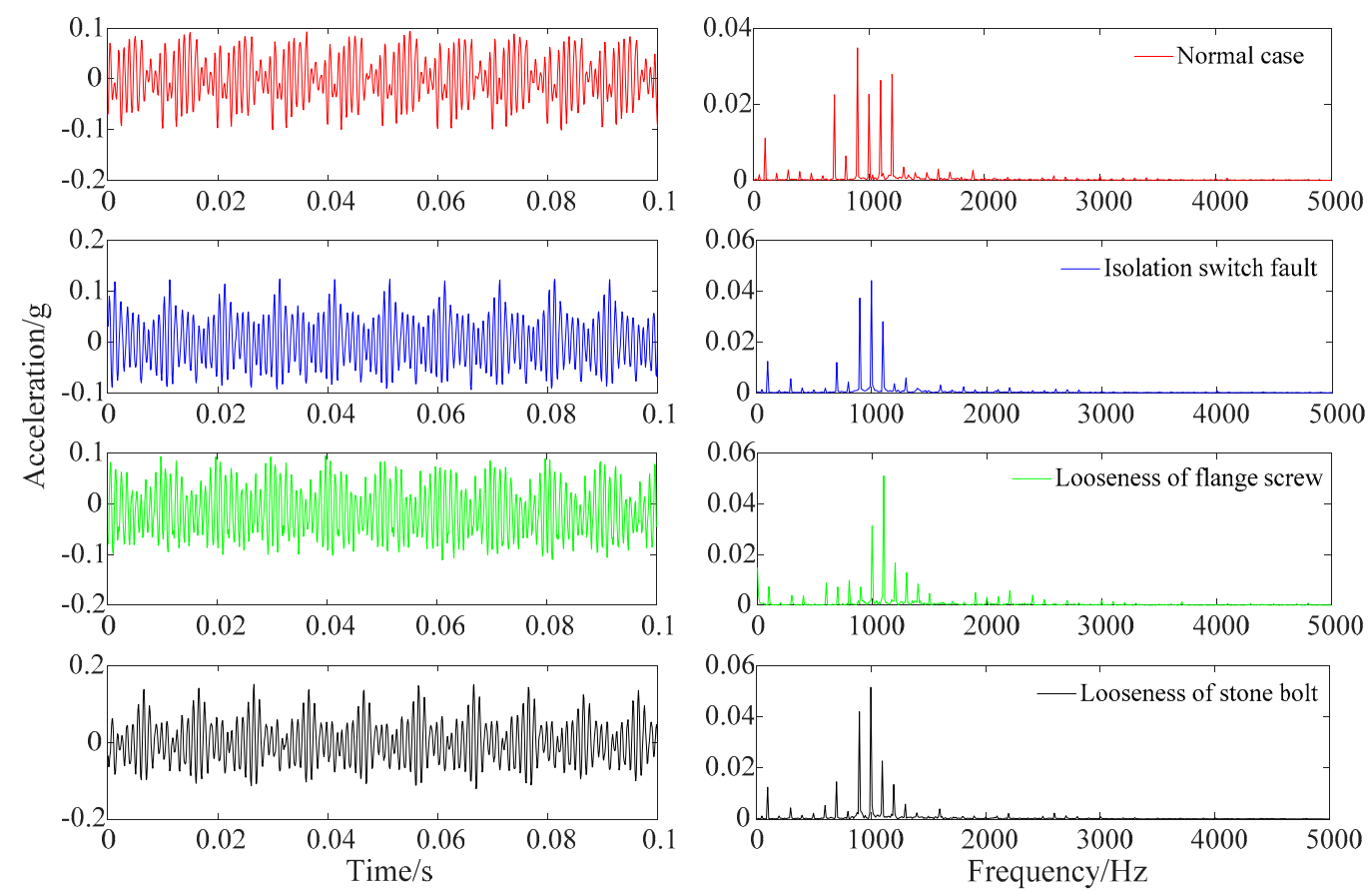

Figure 2. Vibration signal and its spectrum in different working conditions.

The main energy of the vibration signal is concentrated at the frequency around $1 \mathrm{kHz}$, and there is less energy at the frequency of $0.1 \mathrm{kHz}$. The reason is that the GIS system exhibits strong nonlinearity, and its natural frequency is about $1 \mathrm{kHz}$ excited by the electromagnetic force of $100 \mathrm{~Hz}$. When the mechanical fault occurs to GIS, the change of natural frequency leads to differences in spectrum under the same excitation, which are illustrated by the following remarkable differences: (1) the normal case, the isolation switch fault, the looseness of flange screw and the looseness of stone bolt have the highest frequency of vibration signal energy at $0.9 \mathrm{kHz}, 1 \mathrm{kHz}, 1.1 \mathrm{kHz}$ and $1 \mathrm{kHz}$, respectively; (2) the energy ratio of $0.7 \mathrm{kHz}$ and $1.2 \mathrm{kHz}$ in fault working conditions is less than that of the normal case; (3) the energy at $2 \mathrm{kHz}$ of vibration signal in flange fault is higher than that of other working conditions. In order to further analyze the frequency domain characteristics of vibration signals in GIS, three samples were taken from the vibration signals collected in each working conditions to perform a Fourier transform to obtain the spectrum, as shown in Figure 3. 


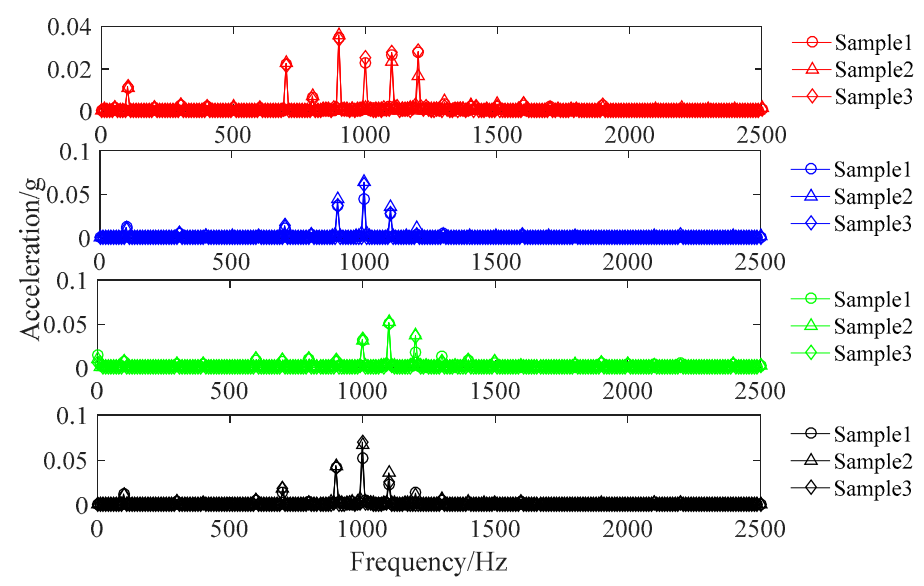

(a) Spectrum analysis of $0-2500 \mathrm{~Hz}$

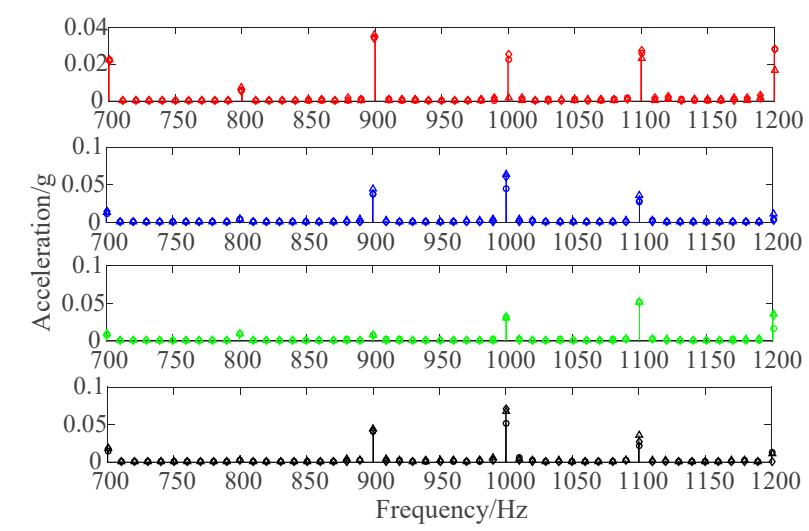

(b) Spectrum analysis of $700-1200 \mathrm{~Hz}$

Figure 3. Spectrum analysis of vibration signals in different working conditions.

It is observed that there is almost no energy distribution at the frequency point above $2500 \mathrm{~Hz}$, so the spectrum range is set below $2500 \mathrm{~Hz}$, as shown in Figure 3a. Due to the serious overlap of frequency points, the $700-1200 \mathrm{~Hz}$ frequency spectrum with more concentrated energy is further selected for observation, as shown in Figure 3b. Figures 2 and 3 indicate the following conclusions: (1) The signal spectrum in the same working condition has a very high similarity, and the energy distribution at most frequency points is basically the same; (2) there is a great difference in the energy distribution at frequency points between vibration signals in different working conditions; (3) the vibration signals have energy distribution at most frequency points, and numerous frequency points with low energy cannot be ignored. The above characteristics can be used to identify the fault types of signals in different working conditions.

\section{Feature Extraction Method of Vibration Signals}

\subsection{Principle of $C F$}

In the field of signal processing, $\mathrm{CF}$ is commonly used to measure the degree of linear correlation between two signals in each frequency component. In this paper, the $\mathrm{CF}$ is used for feature extraction.

Suppose there are two time-domain signals $S_{x}(t)$ and $S_{y}(t)$, the calculation methods of CF are as follows [38]: (1) calculate Fourier spectrum $A_{x}(f)$ and $A_{y}(f)$ of $S_{x}(t)$ and $S_{y}(t)$, respectively; (2) calculate self-power spectral density functions $S_{x}(f)$ and $S_{y}(f)$,

$$
\left\{\begin{array}{l}
S_{x}(f)=A_{x}(f) A_{x}^{*}(f) \\
S_{y}(f)=A_{y}(f) A_{y}^{*}(f)
\end{array}\right.
$$


where $A_{x}{ }^{*}(f)$ and $A_{y}{ }^{*}(f)$ are the complex conjugation of $A_{x}(f)$ and $A_{y}(f)$, respectively; (3) calculate the cross power spectral density function,

$$
S_{x y}(f)=A_{y}(f) A_{x}^{*}(f)
$$

(4) calculate the CF of $S_{x}(t)$ and $S_{y}(t)$,

$$
C_{x y}(f)=\frac{\left|S_{x y}(f)\right|^{2}}{S_{x}(f) S_{y}(f)}
$$

The range of $C_{x y}(f)$ is $[0,1]$, and the larger the value of $C_{x y}\left(f_{0}\right)$ at a certain frequency $f_{0}$, the greater the coherence of signal $S_{x}(t)$ and $S_{y}(t)$ at the frequency of $f_{0}$. $S_{x}(t)$ and $S_{y}(t)$ are irrelevant when CF is 0 and completely coherent when CF is 1 . There are two advantages of the CF: (1) CF can describe the frequency commonality of two signals; (2) CF is not affected by absolute amplitude of the signals and describes the amplitude similarity of two signals at the same frequency point.

Firstly, two groups of samples are taken out from the normal signals for CF calculation, and the results are shown in Figure 4a. Then, a group of samples are extracted from the vibration signals of the normal case and the isolation switch fault respectively for coherence analysis, and the results are shown in Figure 4b.
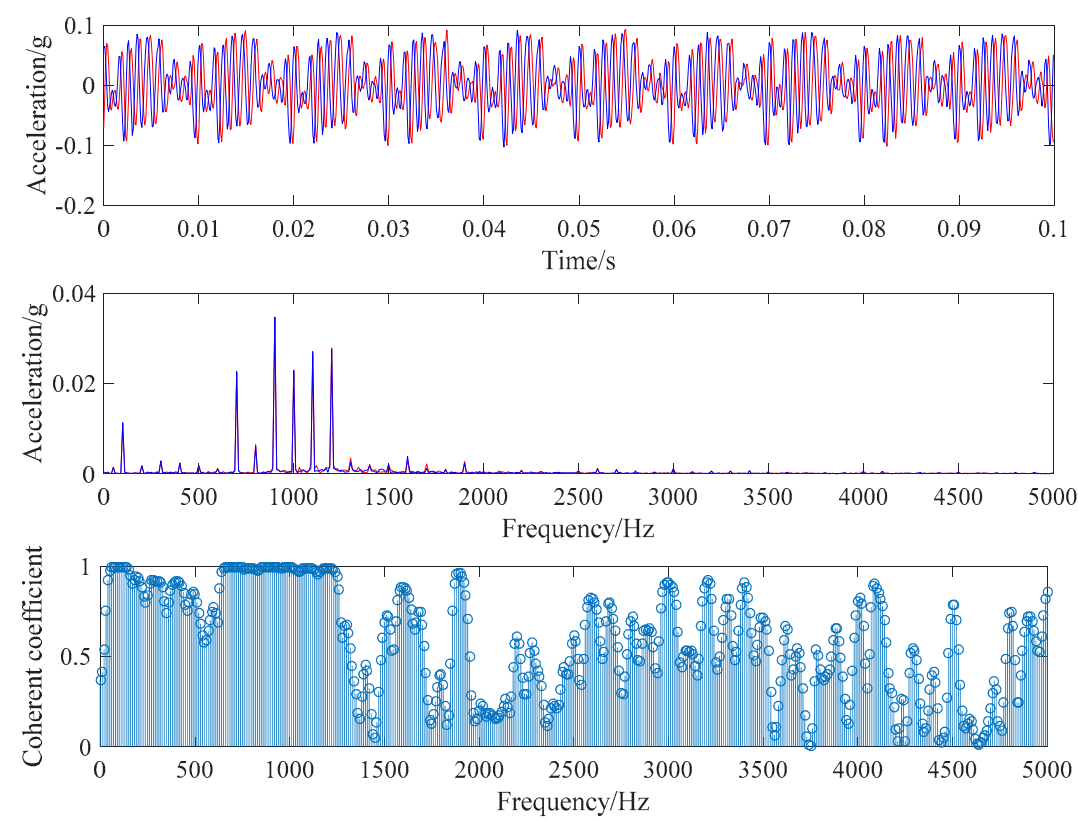

(a) Coherent results of two groups of samples under normal case

Figure 4. Cont. 

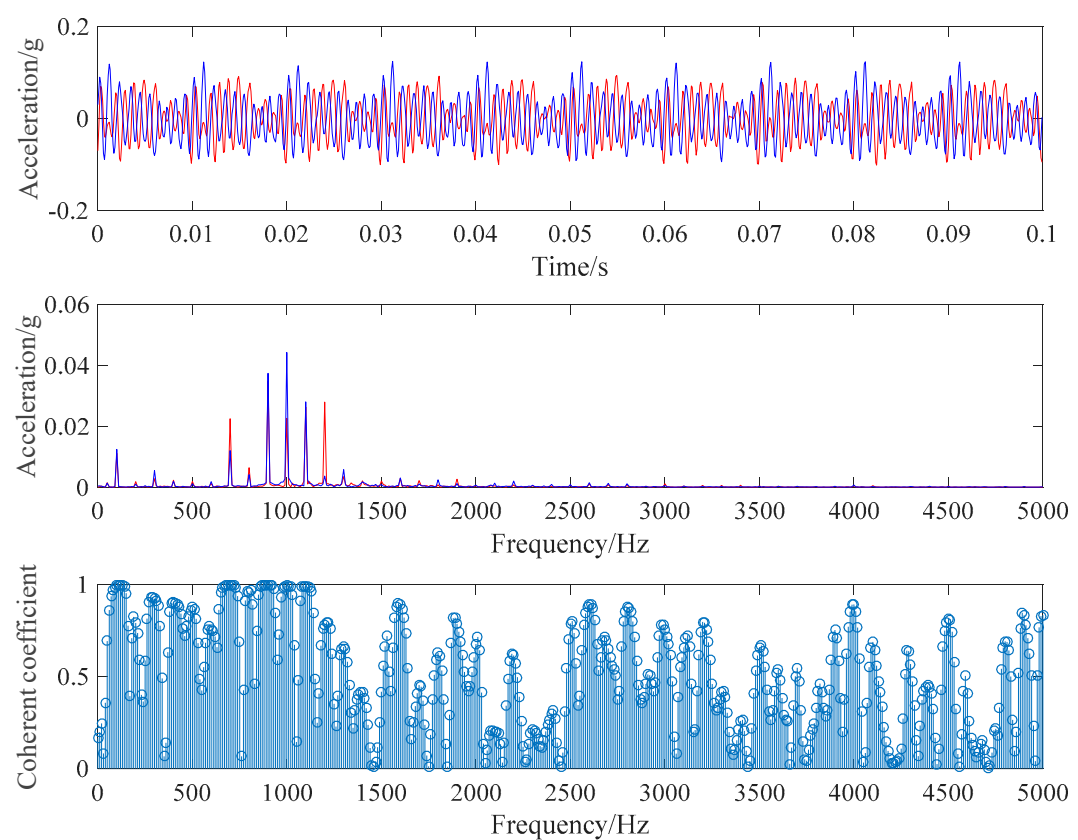

(b) Coherent results of a group of samples under normal case and a group of samples under isolation switch fault

Figure 4. Comparison of coherent results.

As illustrated in Figure 4a, the waveforms of the vibration signals of the two groups of normal samples are basically the same, while the waveforms of the two groups of signals in Figure $4 \mathrm{~b}$ are quite different (red is the normal case sample, blue is the isolation switch fault sample). The energy distribution of the two samples is consistent at most frequency points in the same working condition and has a large difference in different working conditions. The CF of two samples is calculated at the frequency points which are multiples of $10 \mathrm{~Hz}$. The results show that the coherence coefficients of many frequency points are close to 1 in the same working condition, while the frequency points with coherence coefficients close to 1 in different working conditions being few in number.

\subsection{Design Ideas of Feature Construction}

Based on the coherence analysis above, the relationship between two signals at a specific frequency point can be described by the coherent coefficient between signals, thereby obtaining the relevant frequency points describing the commonality of the two signals. However, considering the dispersion of vibration signals and noise interference, it is necessary to calculate the coherence of a large number of signals of the same working condition. This paper designs a feature extraction method based on vibration information in different mechanical conditions, and the specific process is shown in Figure 5. 


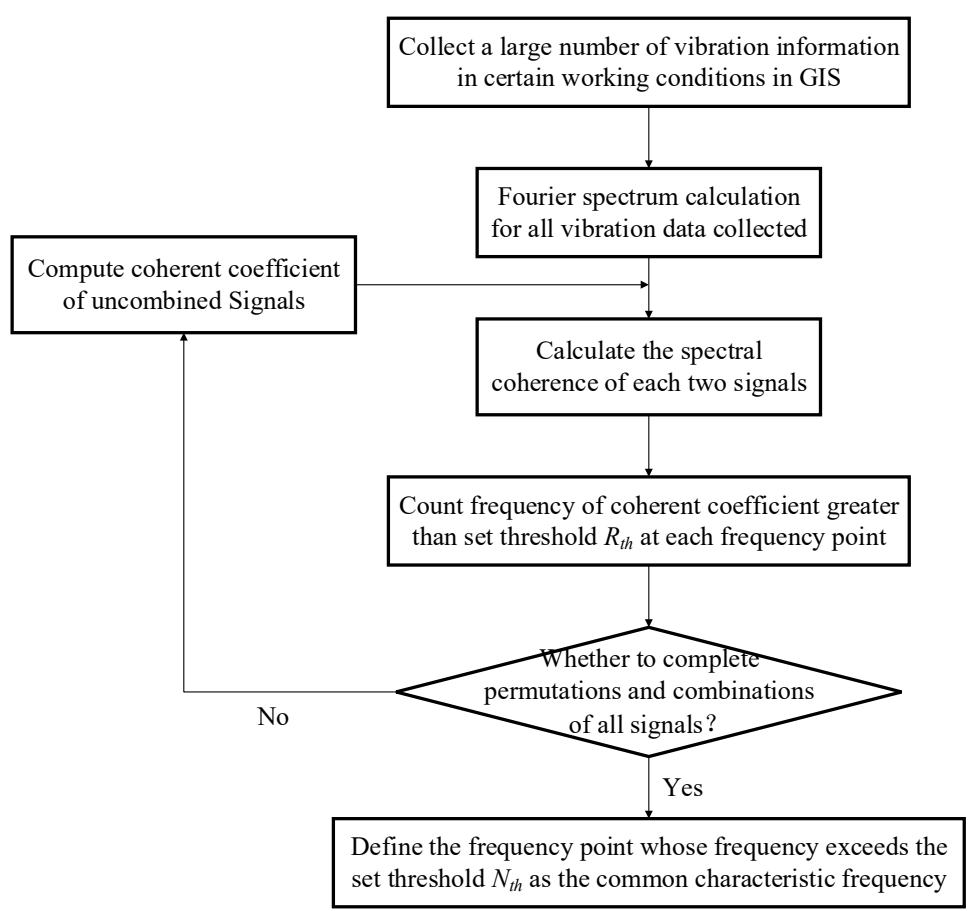

Figure 5. Feature extraction process.

Step 1: Collect $m$ groups of vibration signal samples of a typical type of mechanical defect, and perform a Fourier transform on each sample;

Step 2: Calculate the CF of each of two samples, set the strong correlation threshold $R_{t h}$, judge the coherence coefficient of the selected frequency points (multiples of $10 \mathrm{~Hz}$ ) and the threshold $R_{t h}$, and define the frequency point whose coherence coefficient is larger than $R_{t h}$ as the potential common characteristic frequency feature of this kind of working condition;

Step 3: Count the number of occurrences of each potential common frequency points, set the frequency threshold $N=N_{t h} \times C_{m}^{2}$, where $N_{t h}$ is the frequency threshold coefficient $\left(N_{t h} \in[0,1]\right)$, and define the potential common frequency points whose occurrence times are greater than the threshold $\mathrm{N}$ as the clear common frequency points of the certain working condition to build feature space.

In this paper, the sample number $m$ is 200 , the strong correlation threshold $R_{t h}$ is 0.9 , and the frequency threshold coefficient $N_{t h}$ is 0.65 . The coherent results of each working condition are shown in Figure 6a: (1) the characteristic frequency points above $1.5 \mathrm{kHz}$ and around $0.4 \mathrm{kHz}$ exist only in the flange loosening fault; (2) compared with the fault conditions, there are characteristic frequency points of about $0.5 \mathrm{kHz}$ in the normal condition, but not characteristic frequency points around $0.3 \mathrm{kHz}$; (3) compared with other conditions, the condition of stone bolts loosening lacks the characteristic frequency points near $0.8 \mathrm{kHz}$; (4) only the flange loosening and the stone bolts loosening fault have characteristic frequency points near $0.6 \mathrm{kHz}$. 

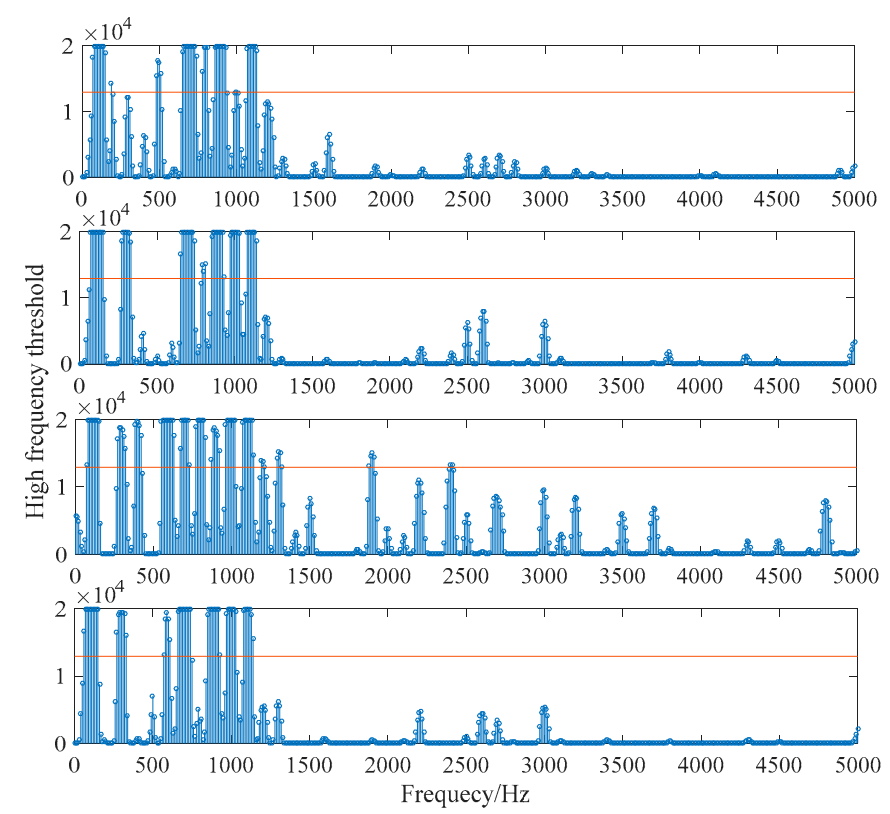

(a) Coherence spectrum statistics.

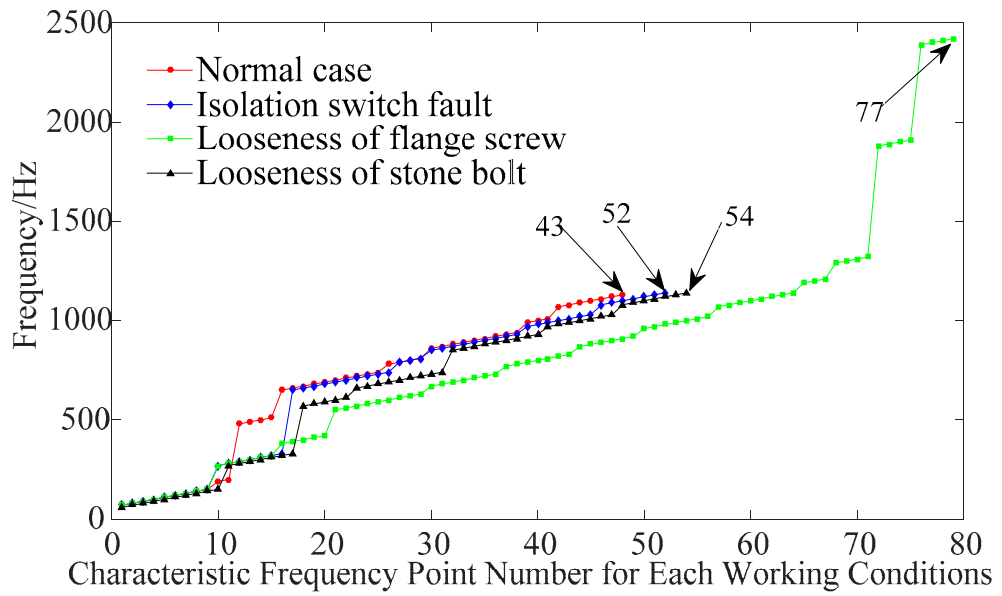

(b) Feature frequency points of different faults.

Figure 6. Characteristic distribution under different faults.

Figure $6 \mathrm{~b}$ shows the characteristic frequency curves in different working conditions. The feature vector and the number of characteristic frequency points were quite different, and the union of feature points was used as the final feature to diagnose fault types.

\section{GIS Fault Diagnosis Method Based on SVM and OCSVM}

\subsection{SVM}

SVM is a method to realize the idea of structural risk minimization. The sample space is linearly partitioned by the optimal classification hyperplane. However, the problem of linearly indivisibility is often encountered in practical problems. Therefore, the data samples need to be mapped into high-dimensional feature space through non-linear transformation, so that it can be transformed into a linear separable problem. Its classification principle is shown in Figure 7. 


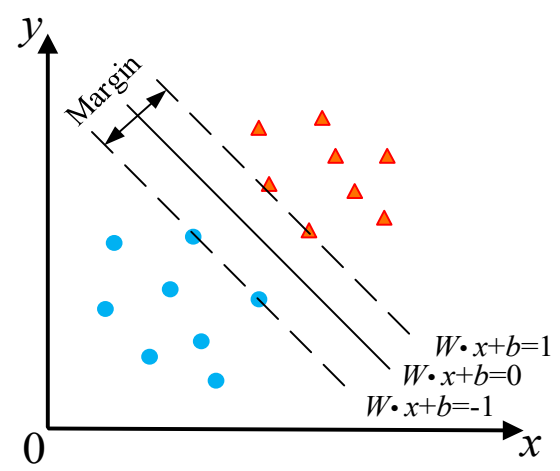

Figure 7. The classification principle of SVM.

For classification of two classes, suppose that the training set of $\mathrm{n}$ samples is $D=\left\{\left(x_{i}, y_{i}\right) \mid i=1,2, \cdots, n\right\}, x_{i} \in R^{n}, y_{i} \in\{-1,+1\}$, then the optimal classification hyperplane $H$ [39] can be expressed as:

$$
W \cdot x+b=0
$$

where $W$ is the normal vector of the optimal classification hyperplane, and $b$ is the constant term.

Two standard hyperplanes $H_{1}(W \cdot x+b=+1)$ and $H_{2}(W \cdot x+b=-1)$ are defined, which are planes through the samples closest to the hyperplane and parallel to the classification hyperplane. In order to maximize the classification interval of hyperplanes, the classification hyperplane is constructed by the following formula to correctly classify all samples [40]:

$$
\left\{\begin{array}{l}
\min _{W} \frac{\|W\|^{2}}{2}=\min _{W} \frac{1}{2} W^{T} W \\
\text { s.t. } y_{i}\left(W \cdot x_{i}+b\right)-1 \geq 0, i=1,2, \cdots, n
\end{array}\right.
$$

Using Lagrange function to solve the above formula, the dual problem of the original problem is described as follows:

$$
\left\{\begin{array}{l}
\max _{a} Q(\alpha)=\sum_{i=1}^{n} \alpha_{i}-\frac{1}{2} \sum_{i=1}^{n} \sum_{j=1}^{n} \alpha_{i} \alpha_{j} y_{i} y_{j}\left(x_{i}^{T} x_{j}\right) \\
\text { s.t. } \sum_{i=1}^{n} \alpha_{i} y_{i} \geq 0, \alpha_{i} \geq 0
\end{array}\right.
$$

where $\alpha_{i}(i=1,2, \cdots, n)$ are Lagrange multipliers, and the maximization of $Q(\alpha)$ depends on the training set $\left\{x_{\mathrm{i}}^{\mathrm{T}} x_{j}\right\}$ and $\left\{y_{i} y_{j}\right\}$. If $\alpha_{i}^{*}$ is the optimal Lagrange multiplier, the optimal hyperplane function is:

$$
f(x)=\operatorname{sgn}\left(\sum_{i=1}^{n} \alpha_{i}^{*} y_{i}\left(x_{i}^{T} x\right)+b\right)
$$

where $x$ is test data.

\subsection{OCSVM}

OCSVM is an anomaly detection algorithm based on machine learning. Unlike traditional SVM, OCSVM only needs one class of samples to train an anomaly detection model, which maps training data to high-dimensional feature space through kernel function and solves an optimal hyperplane in feature space to achieve maximum separation between target data and coordinate origin, as shown in Figure 8. 


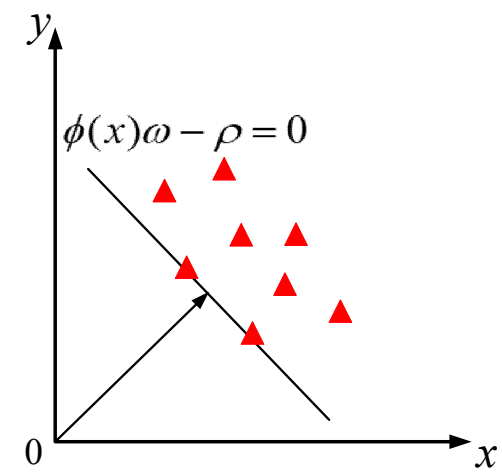

Figure 8. The classification principle of one-class support vector machines (OCSVM).

The minimum objective function sought by OCSVM [41] can be described as:

$$
\left\{\begin{array}{l}
\min \frac{1}{2}\|\omega\|^{2}+\frac{1}{v l} \xi_{i}-\rho \\
\text { s.t. } f(x)=\phi\left(x_{i}\right) \omega \geq \rho-\xi_{i}, \xi_{i} \geq 0
\end{array}\right.
$$

where $i$ is the number of training samples, $x_{i}$ is the sample data, $l$ is the number of training samples, $\phi\left(x_{i}\right)$ is the map of the original space to the feature space, $\omega$ and $\rho$ are the normal vectors of the hyperplane required in the feature space and compensation, respectively, the adjustable parameter $v \in(0,1)$ is the upper limit of the proportion of error samples in the total sample and the relaxation variable $\xi_{i}$ is the degree to which some error samples are misclassified.

The Lagrange function is introduced to get the following formula:

$$
L_{P}=\frac{1}{2}\|\omega\|^{2}+\frac{1}{v l} \xi_{i}-\rho-\sum_{i=1}^{l} \xi_{i} \beta_{i}-\sum_{i=1}^{l}\left(\phi\left(x_{i}\right) \omega-\rho+\xi_{i}\right) \alpha_{i}
$$

where $\alpha_{i}$ and $\beta_{i}$ are Lagrange factors, and the dual problem is obtained by mapping the sample space to the feature space through the Gauss kernel function [41].

$$
\left\{\begin{array}{c}
K\left(x_{i}, x_{j}\right)=<\phi\left(x_{i}\right), \phi\left(x_{j}\right)>=\exp \left(-\mathrm{g}\left\|x_{i}-x_{j}\right\|^{2}\right) \\
\min L=\frac{1}{2} \sum_{i=1}^{l} \sum_{j=1}^{l} \alpha_{i} \alpha_{j} K\left(x_{i}, x_{j}\right), \text { s.t. } 0 \leq \alpha_{i} \leq \frac{1}{v l}
\end{array}\right.
$$

The analytical formula of $\rho$ is obtained by solving Equation (10),

$$
\rho=\sum_{i=1}^{l} \alpha_{i} K\left(x_{i}, x_{j}\right)
$$

The way to find the optimal hyperplane and get the OCSVM based anomaly detection model is represented as Equation (12):

$$
f(x)=\operatorname{sgn}\left(\sum_{i=1}^{l} \alpha_{i} K\left(x_{i}, x_{j}\right)-\rho\right)
$$

For training data $x, f(x)$ indicates that it is positively or negatively located in the hyperplane in high-dimensional space. $f(x)$ is a positive number and $x$ belongs to a normal class; $f(x)$ is a negative number and $x$ belongs to an abnormal class. Therefore, OCSVM can identify non-target samples more accurately. 


\subsection{Fault Diagnosis Process}

The mechanical fault diagnosis technology of GIS proposed in this paper includes two parts: feature extraction and fault diagnosis, and fault diagnosis is divided into state detection and fault recognition. In state detection, the first OCSVM classifier is used to distinguish normal from abnormal cases and solve the problem of unbalance between normal and fault data samples in actual detection. The second OCSVM classifier is used to distinguish known faults from unknown faults and solve the problem of misdiagnosis of unknown faults. In fault recognition, the fault types are judged by an SVM classifier. The specific diagnosis process is shown in Figure 9.

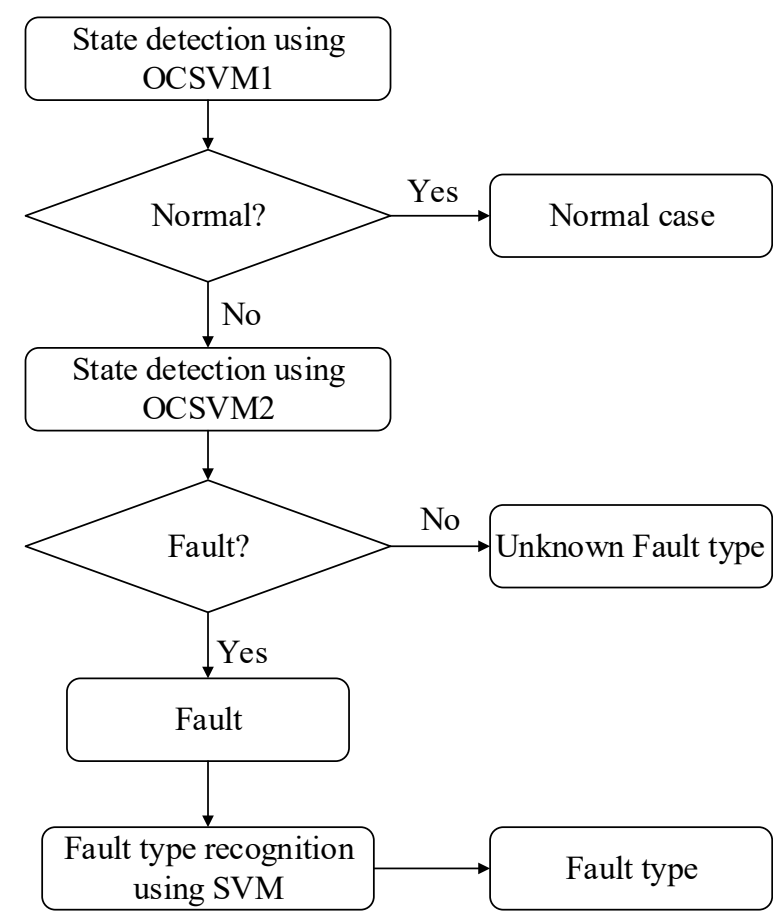

Figure 9. Fault diagnosis process.

Firstly, 70\% of the samples in a normal working condition are randomly selected as training set $A$ for OCSVM1 training; 70\% of the samples in each known type of fault are randomly selected as training set $B$ for OCSVM2 training and SVM training; all remaining samples are used as test set $C$ for evaluating the performance of the diagnosis model.

Secondly, the data of test set $C$ is diagnosed by the OCSVM1 model. The signal with the normal test result is regarded as set $C_{1}$, and the signal with the abnormal result is taken as the set $C_{2}$.

Then, the $C_{2}$ set is diagnosed by the OCSVM2 model. The signals which are detected as faults are regarded as set $C_{3}$ and other data is taken as unknown fault set $C_{4}$.

Lastly, input $C_{3}$ set into the SVM diagnosis model for fault type identification.

\section{Diagnosis Results and Analysis}

\subsection{Discussion of Parameters}

$R_{t h}$ and $N_{t h}$ are two very important parameters in the mechanical fault diagnosis technology proposed in this paper, which jointly determine the feature space of different working conditions, and then affect the final diagnosis results.

If $R_{t h}$ is too small, the difference in frequency energy distribution between signals cannot be effectively distinguished, and it will cause excessive characteristic frequency points, which make it difficult to select characteristic frequency points. If $R_{t h}$ is too large, the effect of dispersion between signals is ignored. 
If $N_{t h}$ is too small, the characteristic frequency points will spread throughout the frequency domain, and the meaning of feature extraction is lost. If $N_{t h}$ is too large, too few characteristic frequency points will reduce the accuracy of fault diagnosis.

In order to discuss the values of parameters $R_{t h}$ and $N_{t h}$, this paper selected [0.5, 0.95] as the value range of $R_{t h},[0.6,0.95]$ as the range of $N_{t h}$ and 0.025 as the step size. Figure 10 shows the relationship between diagnosis accuracy and two key parameters. The diagnosis accuracy is low when both $R_{t h}$ and $N_{t h}$ take large or small values. When values of $R_{t h}$ and $N_{t h}$ are 0.65 and 0.9 , respectively, the diagnosis accuracy is the highest, which reaches $98.75 \%$.

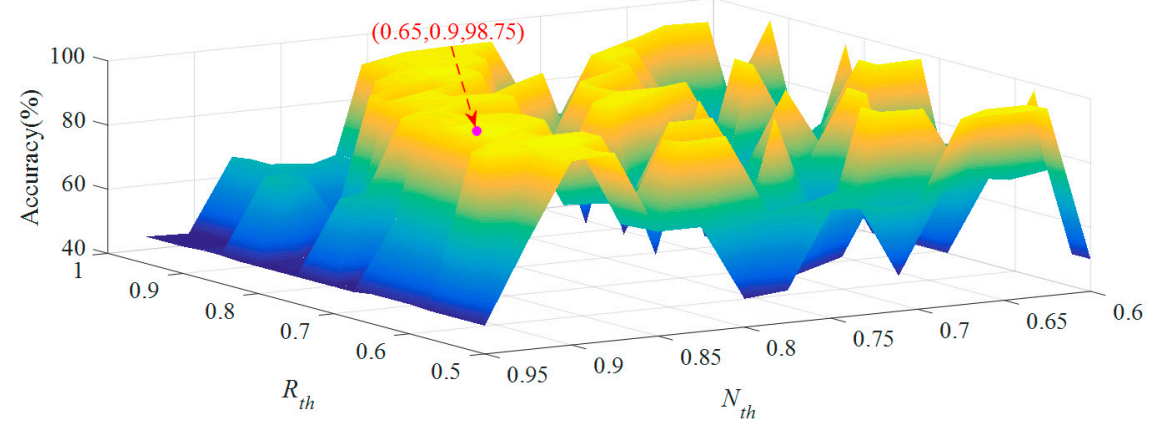

Figure 10. Relationship between accuracy and two key parameters.

In addition to $R_{t h}$ and $N_{t h}$, the main parameters of the diagnosis model using radial basis function (RBF) [42] kernel function are listed in Table 3.

Table 3. Parameters of the proposed method.

\begin{tabular}{cc}
\hline Description & Value \\
\hline gamma of radial basis function (RBF) in & 0.0217 \\
OCSVM1 & 0.66 \\
nu of RBF in OCSVM1 & 93 \\
totalSV in OCSVM1 & 92.3991 \\
rho in OCSVM1 & 0.02 \\
gamma of RBF in OCSVM2 & 0.04 \\
nu of RBF in OCSVM2 & 17 \\
totalSV in OCSVM2 & 16.7936 \\
rho in OCSVM2 & 0.0003 \\
BoxConstraint in SVM (support vector & 1000 \\
machine) & 0.001 \\
CacheSize in SVM & 0.5 \\
DeltaGradientTolerance in SVM & \\
nu of RBF in SVM &
\end{tabular}

\subsection{Diagnosis Results}

After the parameters are determined, 10 experiments were conducted to test the stability of the diagnosis model, and the results are compared with the methods of Softmax, SVM, BPNN and NB. Figure 11 shows the confusion matrix obtained from the first experiment by the proposed method. The diagnosis accuracy of the normal case, isolation switch fault, looseness of flange screw and looseness of stone bolt are $100 \%, 100 \%, 97.5 \%$ and $100 \%$, respectively. The 0 sample is diagnosed as unknown faults. 


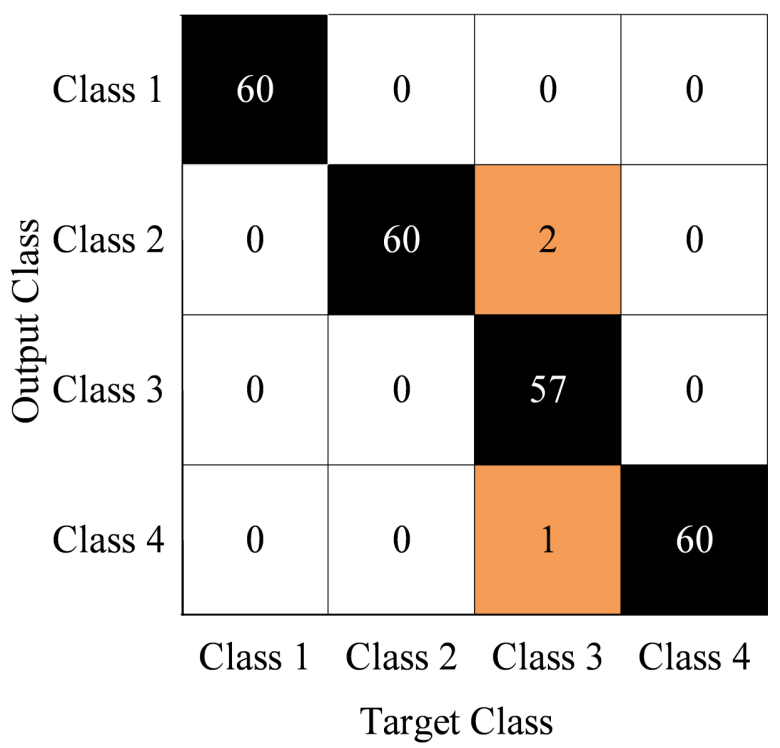

Figure 11. First diagnosis results (confusion matrix).

Figure 11 shows that the proposed method performs well in the first experiment. In order to compare different methods, Tables 4 and 5 show the diagnosis accuracy and F-measure of different methods in the first experiment. Figure 12 shows the comparison of different methods in terms of accuracy and F-measure.

Table 4. Accuracy of the first experiment with different methods.

\begin{tabular}{cccccc}
\hline & \multicolumn{5}{c}{ Accuracy (\%) } \\
\cline { 2 - 6 } Test Method & Normal Case & $\begin{array}{c}\text { Isolation } \\
\text { Switch Fault }\end{array}$ & $\begin{array}{c}\text { Looseness of } \\
\text { Flange Screw }\end{array}$ & $\begin{array}{c}\text { Looseness of } \\
\text { Stone Bolt }\end{array}$ & $\begin{array}{c}\text { All Conditions } \\
\text { Softmax }\end{array}$ \\
SVM & 81.667 & 65.000 & 70.000 & 81.667 & 74.583 \\
\hline Back propagation neural & 85.000 & 71.667 & 75.000 & 70.000 & 75.417 \\
networks (BPNN) & 81.667 & 70.000 & 76.667 & 76.667 & 76.250 \\
Naive Bayes (NB) & 81.667 & 86.667 & 85.000 & 86.667 & 85.000 \\
OCSVM+SVM & 100.000 & 100.000 & 97.500 & 100.000 & 98.75 \\
\hline
\end{tabular}

Table 5. F-measure of first experiment with different methods.

\begin{tabular}{cccccc}
\hline \multirow{2}{*}{ Test Method } & \multicolumn{5}{c}{ F-measure (\%) } \\
\cline { 2 - 6 } & Normal Case & $\begin{array}{c}\text { Isolation } \\
\text { Switch Fault }\end{array}$ & $\begin{array}{c}\text { Looseness of } \\
\text { Flange Screw }\end{array}$ & $\begin{array}{c}\text { Looseness of } \\
\text { Stone Bolt }\end{array}$ & All Conditions \\
\hline Softmax & 77.778 & 69.643 & 71.765 & 78.400 & 74.396 \\
SVM & 85.000 & 72.269 & 72.581 & 71.795 & 75.411 \\
BPNN & 81.667 & 67.742 & 78.632 & 77.311 & 76.338 \\
NB & 85.217 & 85.950 & 85.000 & 83.871 & 85.010 \\
OCSVM+SVM & 100.000 & 98.360 & 97.436 & 99.174 & 98.742 \\
\hline
\end{tabular}




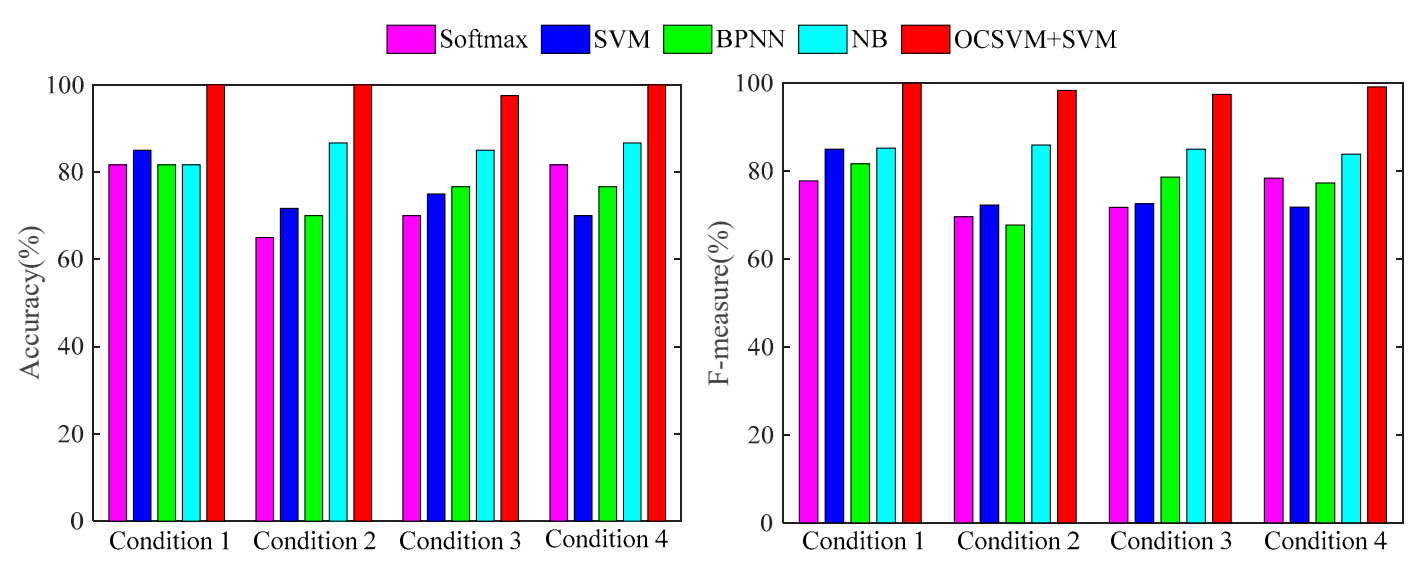

Figure 12. Accuracy and F-measure of first test with different methods.

Tables 4 and 5, and Figure 12 exhibit that the accuracy and F-measure of the proposed method are higher than other classification methods in each working condition, which shows that the method is more suitable than standard deep learning for feature learning in the paper. The results of 10 random experiments are shown in Figure 13.

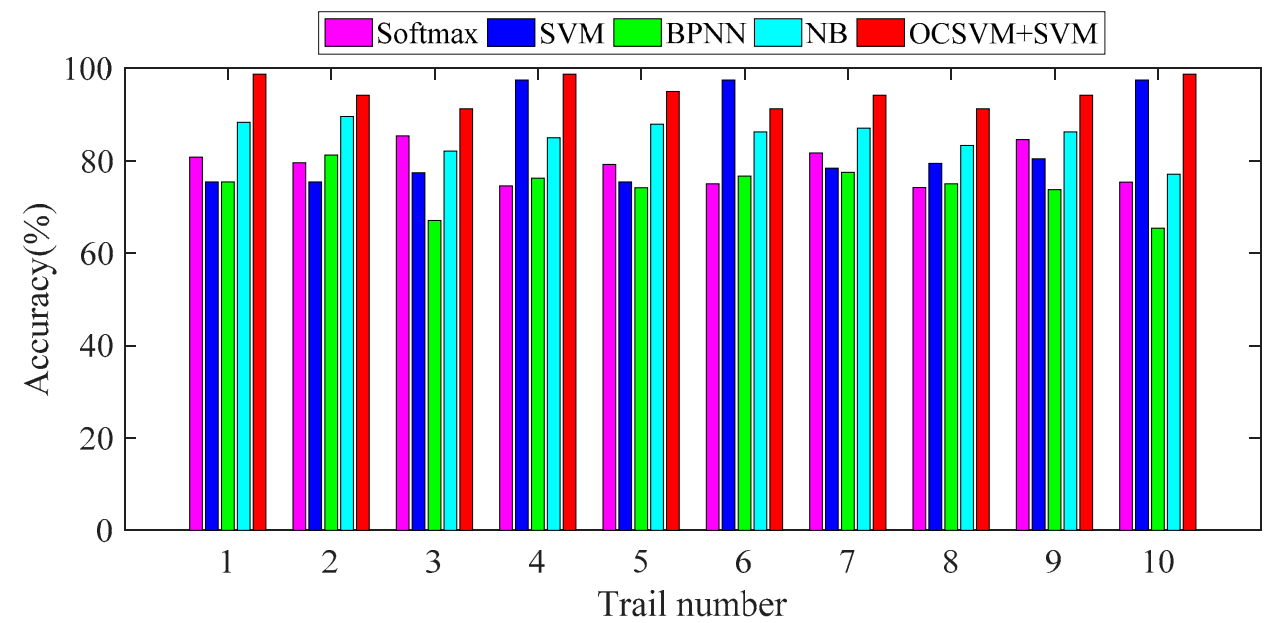

Figure 13. Diagnosis results of 10 tests with different methods.

In 10 random experiments, the diagnosis accuracy of the proposed method is above $90 \%$, which is generally higher than Softmax, SVM, BPNN and NB. It is only slightly inferior to SVM in the Test 6 test, which indicates that the method proposed in this paper is more stable and has a better diagnosis effect.

In order to further evaluate the diagnosis model, the average and standard deviation were used as indicators to compare the diagnosis accuracy of each working condition with different methods. The statistical results are shown in Figure 14 and Table 6. 


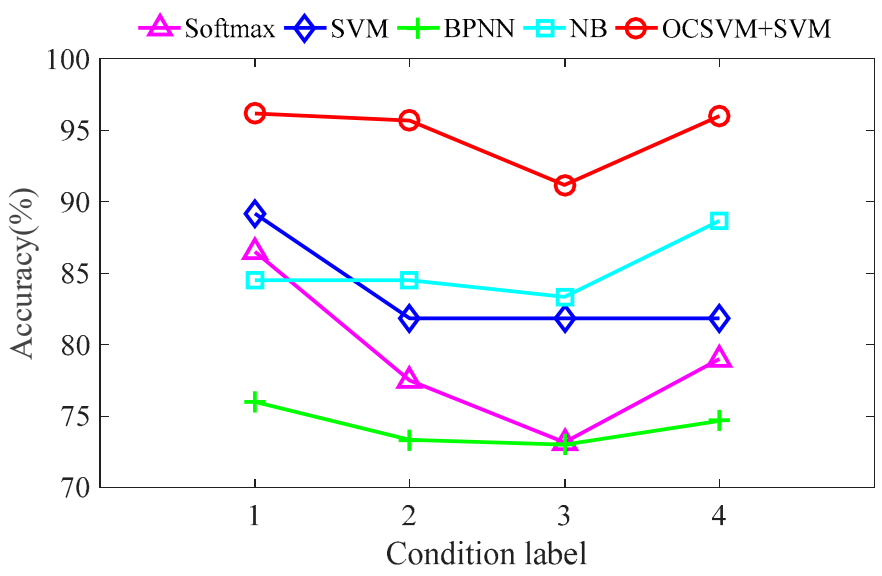

Figure 14. Comparison of diagnosis mean values under different working conditions.

Table 6. Diagnosis results (average and standard deviation).

\begin{tabular}{cccccc}
\hline \multirow{3}{*}{ Test Method } & \multicolumn{5}{c}{ Mean and Standard Deviation of Accuracy (\%) } \\
\cline { 2 - 6 } & Normal Case & $\begin{array}{c}\text { Isolation } \\
\text { Switch Fault }\end{array}$ & $\begin{array}{c}\text { Looseness of } \\
\text { Flange Screw }\end{array}$ & $\begin{array}{c}\text { Looseness of } \\
\text { Stone Bolt }\end{array}$ & All Conditions \\
\hline Softmax & $86.500 \pm 6.007$ & $77.500 \pm 6.538$ & $73.167 \pm 5.119$ & $79.000 \pm 8.285$ & $79.046 \pm 4.147$ \\
SVM & $89.167 \pm 6.249$ & $81.833 \pm 10.045$ & $81.833 \pm 11.988$ & $81.833 \pm 13.259$ & $83.444 \pm 9.847$ \\
BPNN & $76.000 \pm 5.784$ & $73.333 \pm 6.894$ & $73.000 \pm 4.360$ & $74.667 \pm 7.106$ & $74.254 \pm 4.727$ \\
NB & $84.500 \pm 5.215$ & $84.500 \pm 3.853$ & $83.333 \pm 3.514$ & $88.667 \pm 4.216$ & $85.290 \pm 3.670$ \\
OCSVM+SVM & $96.167 \pm 4.648$ & $95.667 \pm 2.808$ & $91.167 \pm 2.727$ & $96.000 \pm 3.443$ & $94.751 \pm 3.088$ \\
\hline
\end{tabular}

Figure 14 and Table 5 show that: (1) the average diagnosis accuracy of the proposed classifier is higher than that of other methods and the standard deviation is lower than that of other methods, which show that the proposed classifier is more stable and reliable; (2) considering the standard deviation, the diagnosis accuracy of the proposed classifier may be lower than other methods, but it is obviously superior to other methods overall, which shows that the method proposed in this paper is more effective for the classification of mechanical working conditions in GIS, and the diagnosis model is more reliable; (3) the feature extraction method proposed in this paper is effective and feasible.

To verify the ability of the diagnosis model to judge the unknown faults, the 20 groups of vibration samples of two composite faults (a combination of isolation switch fault and looseness of stone bolt, a combination of looseness of flange screw and looseness of stone bolt) were collected, respectively. Diagnosis results are shown in Table 7.

Table 7. Diagnosis results of two composite faults.

\begin{tabular}{cccccc}
\hline Actual Working Condition & Normal Case & $\begin{array}{c}\text { Isolation } \\
\text { Switch Fault }\end{array}$ & $\begin{array}{c}\text { Looseness of } \\
\text { Flange Screw }\end{array}$ & $\begin{array}{c}\text { Looseness of } \\
\text { Stone Bolt }\end{array}$ & $\begin{array}{c}\text { Unknown } \\
\text { Fault Type }\end{array}$ \\
\cline { 2 - 6 } & 0 & 2 & 0 & 1 & 17 \\
\hline $\begin{array}{c}\text { Isolation switch fault and } \\
\text { looseness of stone bolt } \\
\begin{array}{c}\text { Looseness of flange screw and } \\
\text { looseness of stone bolt }\end{array}\end{array}$ & 0 & 0 & 1 & 0 & 19 \\
\hline
\end{tabular}

The diagnostic accuracy of the two composite faults are $85 \%$ and $95 \%$, respectively, indicating that the diagnosis model has a good ability to identify unknown faults.

\section{Conclusions}

It is important to improve the reliability of the operation of GIS equipment and find out the potential defects in the operation in time. Thus, a holistic approach composed of a method to extract features and a multi-layer classifier was proposed in this study. First, we developed the characteristic 
description method based on CF. Then, based on OCSVM and SVM, a multi-layer classifier was constructed to conduct fault diagnosis.

The usefulness of feature learning was verified by a comparison among five machine learning methods in a series of experiments. The experimental results indicated that the technique of using CF for feature screening is feasible, and a new idea is provided for feature extraction. At the same time, it also proves that the classifier proposed in the paper is more stable and reliable than other methods. The fault diagnosis method proposed in this paper can play a certain role in the condition detection of GIS and promote the development of intelligent detection technology of the mechanical state in GIS.

Author Contributions: Y.Y. designed the research method; M.S. and Y.Y. wrote the draft; J.B. and L.W. contributed to the experimental section; L.X. gave a detailed revision; W.J. provided important guidance. All authors have read and approved the final manuscript.

Funding: This work is supported by the National Nature Science Foundation of China under Grant 51677002 and China Postdoctoral Science Foundation under Grant 2018M631307.

Conflicts of Interest: The authors declare no conflict of interest.

\section{References}

1. Jo, H.E.; Wang, G.M.; Kim, S.J.; Kil, G.S. Comparison of partial discharge characteristics in SF6 gas under AC and DC. Trans. Electr. Electron. Mater. 2015, 16, 323-327. [CrossRef]

2. Lothar, H.; Franz, B. Compact and reliable—Decades of benefits: Gas-insulated switchgear from 52 to $1100 \mathrm{kV}$. ABB Review. 2009. Available online: https://search-ext.abb.com/library/Download.aspx?DocumentID= 9AKK104295D4901\&LanguageCode=en\&DocumentPartId=\&Action=Launch (accessed on 25 April 2019).

3. Judd, M.D.; Farish, O.; Hampton, B.F. The excitation of UHF signals by partial discharges in GIS. IEEE Trans. Dielectr. Electr. Insul. 1996, 3, 213-228. [CrossRef]

4. Li, T.; Rong, M.; Zheng, C.; Wang, X. Development simulation and experiment study on UHF Partial Discharge Sensor in GIS. IEEE Trans. Dielectr. Electr. Insul. 2012, 19, 1421-1430. [CrossRef]

5. Gomez, F.A.; Albarracin-Sanchez, R.; Vecino, F.G.; Arrabe, R.G. Diagnosis of Insulation Condition of MV Switchgears by Application of Different Partial Discharge Measuring Methods and Sensors. Sensors 2018, 18, 720. [CrossRef]

6. Cosgrave, J.A.; Vourdas, A.; Jones, G.R.; Spencer, J.W.; Murphy, M.M.; Wilson, A. Acoustic monitoring of partial discharges in gas insulated substations using optical sensors. IEE Proc. A Sci. Meas. Technol. 2006, 140, 369-374. [CrossRef]

7. Markalous, S.; Tenbohlen, S.; Feser, K. Detection and location of partial discharges in power transformers using acoustic and electromagnetic signals. IEEE Trans. Dielectr. Electr. Insul. 2008, 15, 1576-1583. [CrossRef]

8. Khan, I.U.; Wang, Z.D.; Cotton, I.; Northcote, S. Dissolved gas analysis of alternative fluids for power transformers. IEEE Electr. Insul. Mag. 2007, 23, 5-14. [CrossRef]

9. Faiz, J.; Soleimani, M. Dissolved gas analysis evaluation in electric power transformers using conventional methods a review. IEEE Trans. Dielectr. Electr. Insul. 2017, 24, 1239-1248. [CrossRef]

10. Wang, G.; Kil, G.S. Measurement and Analysis of Partial Discharge using an Ultra-High Frequency Sensor for Gas Insulated Structures. Metrol. Meas. Syst. 2017, 24, 515-524. [CrossRef]

11. Griffin, D.; Lim, J.S. Signal estimation from modified short-time Fourier transform. IEEE Trans. Acoust. Speech Signal Process. 1984, 32, 236-243. [CrossRef]

12. Dong, Y.; Tang, J.; Zeng, F.; Liu, M. Features Extraction and Mechanism Analysis of Partial Discharge Development under Protrusion Defect. J. Electr. Eng. Technol. 2015, 10, 344-354. [CrossRef]

13. Li, X.; Wang, X.; Xie, D.; Wang, X.Q.; Yang, A.J.; Rong, M.Z. Time-frequency analysis of PD-induced UHF signal in GIS and feature extraction using invariant moments. Iet Sci. Meas. Technol. 2018, 12, 169-175. [CrossRef]

14. Lin, Y.H. Using k-means clustering and parameter weighting for partial-discharge noise suppression. IEEE Trans. Power Deliv. 2011, 26, 2380-2390. [CrossRef]

15. Huang, N.E.; Zheng, S.; Long, S.R. The empirical mode decomposition and the Hilbert spectrum for nonlinear and non-stationary time series analysis. Proc. Math. Phys. Eng. Sci. 1998, 454, 903-995. [CrossRef] 
16. Wu, Z.; Liu, B.; Wu, L.; Ma, S.; Ren, B.; Su, J.T.; Hou, Y. Analysis of GIS Mechanical Vibration Signal Based on EMD Algorithm. In Proceedings of the International Conference on Information Science \& Control Engineering, Beijing, China, 8-10 July 2016; pp. 853-857.

17. Shang, Y.; Liu, Q.; Niu, B.; Zhang, M.H.; Qi, W.D.; Wu, J.F. Mechanical fault diagnosis system based on acoustic feature analysis in gas insulated switchgear. In Proceedings of the International Conference on Electrical Materials \& Power Equipment, Xi'an, China, 14-17 May 2017; pp. 610-615.

18. Yang, J.G.; Liu, Y.; Hu, D.G.; Wu, B.; Che, B.; Li, J.H. Transient electromagnetic force analysis of GIS bus based on FEM. In Proceedings of the International Conference on Condition Monitoring and Diagnosis, Xi'an, China, 25-28 September 2016; pp. 554-557.

19. Yang, J.G.; Liu, Y.; Hu, D.G.; Wu, B.; Li, J.H. Transient vibration study of GIS bus based on FEM. In Proceedings of the Power and Energy Engineering Conference, Xi'an, China, 25-28 October 2016; pp. 1092-1095.

20. Sun, Q.S.; Cao, T.; Hou, Y.; Zhao, T. Detection and Analysis Based on the Abnormal Mechanical Vibration Signal of GIS. In Proceedings of the Fifth International Conference on Instrumentation and Measurement, Computer, Communication and Control, Qinhuangdao, China, 18-20 September 2015; pp. 131-134.

21. Liu, Y.; Jia, Y.Y.; Yang, J.G.; Song, S.Q.; Wu, B.; Li, J.H. Research of mechanical state diagnosis techniques in GIS bus connector based on mechanical vibration. In Proceedings of the 2018 12th International Conference on the Properties and Applications of Dielectric Materials, Xi'an, China, 20-24 May 2018; pp. 682-685.

22. Shen, X.; Lin, Z.; Peng, G.; Tang, S.; Zhang, Y. Research on mechanical fault diagnosis of Ultra high voltage GIS based on the combination of neighbor algorithm and FCM. In Proceedings of the Power and Energy Engineering Conference, Xi'an, China, 25-28 October 2016; pp. 2045-2049.

23. Jeub, M.; Schafer, M.; Esch, T.; Vary, P. Model-Based Dereverberation Preserving Binaural Cues. IEEE Trans. Audio Speech Language Process. 2010, 18, 1732-1745. [CrossRef]

24. Yuan, F.; Xue, X.; Shi, J.; Zhang, L.; Huang, J.F. Holistic learning-based high-order feature descriptor for smoke recognition. Int. J. Wavelets Multiresolut. Inf. Process. 2018, 468, 193-212. [CrossRef]

25. Dai, G.; Xie, J.; Fang, Y. Deep Correlated Holistic Metric Learning for Sketch-Based 3D Shape Retrieval. IEEE Trans. Image Process. 2018, 27, 3374-3386. [CrossRef] [PubMed]

26. Stanev, V.; Oses, C.; Kusne, A.G.; Rodriguez, E.; Paglione, J.; Curtarolo, S.; Takeuchi, I. Machine learning modeling of superconducting critical temperature. Npj Comput. Mater. 2018, 4, 29. [CrossRef]

27. Miltiadis, A.; Barr, E.T.; Premkumar, D.; Charles, S. A survey of machine learning for big code and naturalness. ACM Comput. Surv. 2018, 51, 1-37.

28. Schölkopf, B.; Platt, J.C.; Shawe-Taylor, J.; Smola, A.J.; Williamson, R.C. Estimating the Support of a High-Dimensional Distribution. Neural Comput. 2001, 13, 1443-1471. [CrossRef]

29. Cortes, C.; Vapnik, V. Support-vector networks. Mach. Learn. 1995, 20, 273-297. [CrossRef]

30. Maglaras, L.A.; Jiang, J.; Cruz, T. Integrated OCSVM mechanism for intrusion detection in SCADA systems. Electron. Lett. 2014, 50, 1935-1936. [CrossRef]

31. Jung, D.; Ng, K.Y.; Frisk, E.; Krysander, M. Combining model-based diagnosis and data-driven anomaly classifiers for fault isolation. Control Eng. Pract. 2018, 80, 146-156. [CrossRef]

32. Jung, D.; Ng, K.Y.; Frisk, E.; Krysander, M. A combined diagnosis system design using model-based and data-driven methods. In Proceedings of the 3rd Conference on Control and Fault-tolerant Systems, Barcelona, Spain, 7-9 September 2016; pp. 177-182.

33. Vapnik, V.N. The Nature of Statistical Learning Theory; Springer: New York, NY, USA, 1995.

34. Suykens, J.A.K.; Vandewalle, J. Least Squares Support Vector Machine Classifiers. Neural Process. Lett. 1999, 9, $293-300$. [CrossRef]

35. Bhavish, K.N.; Sharon, M.J.; Nirmala, M. SoftMax based User Attitude Detection Algorithm for Sentimental Analysis. Procedia Comput. Sci. 2018, 125, 313-320.

36. Werbos, P.J. Beyond Regression: New Tools for Prediction And Analysis in The Behavioral Sciences. Ph.D. Thesis, Harvard University, Cambridge, MA, USA, 1974.

37. Zhang, N.N.; Wu, L.F.; Yang, J.; Guan, Y. Naive Bayes Bearing Fault Diagnosis Based on Enhanced Independence of Data. Sensors 2018, 18, 463. [CrossRef]

38. Sarkar, S.; Healey, G. Hyperspectral Texture Synthesis Using Histogram and Power Spectral Density Matching. IEEE Trans. Geosci. Remote Sens. 2010, 48, 2261-2270. [CrossRef] 
39. Yeom, H.G.; Jang, I.H.; Sim, K.B. Variance considered machines: Modification of optimal hyperplanes in support vector machines. In Proceedings of the IEEE International Symposium on Industrial Electronics, Seoul, Korea, 5-8 July 2009; pp. 1133-1136.

40. Wang, L.; Jia, X. Integration of Soft and Hard Classifications Using Extended Support Vector Machines. IEEE Geosci. Remote Sens. Lett. 2009, 6, 543-547. [CrossRef]

41. Xiao, Y.; Wang, H.; Xu, W. Parameter Selection of Gaussian Kernel for One-Class SVM. IEEE Trans. Cybern. 2015, 45, 927-939.

42. Chen, S.; Cowan, C.N.; Grant, P.M. Orthogonal least squares learning algorithm for radial basis function networks. IEEE Trans. Neural Netw. 1991, 64, 829-837. [CrossRef]

(C) 2019 by the authors. Licensee MDPI, Basel, Switzerland. This article is an open access article distributed under the terms and conditions of the Creative Commons Attribution (CC BY) license (http://creativecommons.org/licenses/by/4.0/). 